$\begin{array}{ll}\text { Research Square } & \begin{array}{l}\text { Preprints are preliminary reports that have not undergone peer review. } \\ \text { They should not be considered conclusive, used to inform clinical practice, } \\ \text { or referenced by the media as validated information. }\end{array}\end{array}$

\title{
Clinical Course and Characteristics of COVID-19 in Patients With Inborn Errors of Immunity: A Retrospective Multicenter Experience From Iran
}

\section{Abdollah Karimi}

Shahid Beheshti University of Medical Sciences

Yousef Shokri

Allergist \& Clinical immunologist, Shahid Beheshti University of Medical Sciences, Tehran, Iran

Mahnaz Jamee

Shahid Beheshti University of Medical Sciences https://orcid.org/0000-0002-0732-9170

Atefh Heidari

Shahid Beheshti University of Medical Sciences

Fatemeh Nazarpack

Shahid Beheshti University of Medical Sciences

Mazdak Fallahi

Shahid Beheshti University of Medical Sciences

Reza Shiari

Shahid Behehsti University of Medical Sciences

Niusha Sharifinejad

Alborz University of Medical Sciences

Mehrnaz Mesdaghi

Shahid Beheshti University of Medical Sciences Mitra Khalili

Shahid Beheshti University of Medical Sciences

Shahnaz Armin

Shahid Beheshti University of Medical Sciences

Roxana Mansour Ghanaie

Shahid Beheshti University of Medical Sciences

Sedigheh Rafiei Tabatabaei

Shahid Beheshti University of Medical Sciences

Seyed Alireza Fahimzad

Shahid Beheshti University of Medical Sciences

Narges Eslami

Shahid Beheshti University of Medical Sciences

Samin Sharafian

Shahid Beheshti University of Medical Sciences

Seyed Alireza Mahdaviani

Shahid Beheshti University of Medical Sciences

Seyed Hesamedin Nabavizadeh

Shiraz University of Medical Sciences

Hossein Esmaeilzadeh

Shiraz University of Medical Sciences

Zahra Kanannejad

Shiraz University of Medical Sciences

Maryam Babaei

Shiraz University of Medical Sciences

Mahboubeh Mansouri

Shahid Beheshti University of Medical Sciences

Delara Babaei

Shahid Beheshti University of Medical Sciences Shahrzad Fallah

Shahid Beheshti University of Medical Sciences Nasrin Khakbazan Fard

Shahid Beheshti University of Medical Sciences

Seyedeh Atefeh Hashemimoghaddam 
Shahid Beheshti University of Medical Sciences

Soheila Alyasin

Shiraz University of Medical Sciences

Zahra Chavoshzadeh ( $\nabla$ zahra_chavoshzadeh@yahoo.com )

Shahid Beheshti University of Medical Sciences

\section{Research Article}

Keywords: Inborn errors of immunity, IEI, Primary immunodeficiency, Coronavirus disease 2019, COVID-19, SARS-CoV-2

Posted Date: October 7th, 2021

DOI: https://doi.org/10.21203/rs.3.rs-957348/v1

License: (c) (i) This work is licensed under a Creative Commons Attribution 4.0 International License. Read Full License 


\section{Abstract \\ Purpose}

This study aimed to assess the effect of COVID-19 on patients with IEls, the potentially at-risk population, regarding the clinical course, complications, severity, and outcomes.

\section{Methods}

This two-phase study was conducted on patients from three referral immunodeficiency centers in Iran. At phase one, 98 IEl patients with COVID-19 infection were evaluated by telephone follow-up (TFU). At phase two, the demographic, clinical, and laboratory records of clinically confirmed 33 IEl patients with COVID-19 infection were collected and analyzed.

\section{Results}

At phase one, 16.3\% represented COVID-19 infection without any report of pediatric intensive care unit (PICU) admission or death. During the second phase, combined immunodeficiency (CID) (42.4\%) and predominantly antibody deficiencies (PADs) (33.3\%) were the predominant immune defects. Atopy (27.3\%) and lung disorders $(27.3 \%)$ were the frequent pre-existing comorbidities. Organomegaly $(p=0.030)$ and renal disorders $(p=0.033)$ were significantly associated with the development of respiratory insufficiency. Cyanosis, tachypnea, intercostal retraction, and seizure were the chief complaints of patients who were more likely to progress respiratory insufficiency $(p<0.05)$, being admitted to the PICU $(p<0.05)$, and/or deceased $(p<0.05)$. Laboratory evaluation revealed a marked positive correlation between D-Dimer $(p=0.045)$, prothrombin time $(p=0.045)$, C-reactive protein $(p=0.041)$, proteinuria $(p=0.013)$, ferritin $(p=0.020)$, metabolic acidosis $(p=0.003)$, and troponin ( $p=0.049)$ level with mortality. We detected a significant association between the chest X-ray pattern of COVID-19 infection with PICU admission $(p=0.023)$ and death $(p=0.046)$.

\section{Conclusion}

In the current study, patients with CID and PAD were introduced as patients at high risk of COVID-19 infection, who may need extra protective and therapeutic measurements.

\section{Introduction}

Coronavirus disease 2019 (COVID-19) is a respiratory illness caused by a novel coronavirus, severe acute respiratory syndrome coronavirus 2 (SARS-CoV-2), first diagnosed in December 2019 in Wuhan, China, and introduced as a pandemic in March 2020 by World Health Organization (1). The SARS-CoV-2 virus belongs to $\beta$-Coronaviruses and infects human cells by binding to the angiotensin-converting enzyme 2 , expressed predominantly by lungs and intestinal epithelial cells, alveolar cells, and vascular endothelial cells (2). SARS-CoV-2 spreads within the human population mainly through droplet transmission and as of May 2021, 171,448,311 individuals have been infected with this virus, causing more than 3,714,831 deaths worldwide (3).

Risk factors associated with severe COVID-19 disease include advancing age (especially $>65$ years), obesity (body mass index $>30$ ), diabetes mellitus, chronic pulmonary diseases, cardiovascular diseases, chronic renal diseases, smoking, malignancies, chronic allergic diseases, and primary or secondary immunodeficiency (4). Among others, the clinical impact of the latter risk factor in COVID-19 infection has not been yet precisely determined.

Patients with inborn errors of immunity (IEls) can be considered as prototypes of immune defects. IEls form a heterogeneous group of inherited disorders caused by specific gene mutations that impact immune system development and function (5). To date, more than 430 monogenic IEls have been reported (5, 6) and the global prevalence of IEls has been estimated to be 1:8500-1:100000 for symptomatic patients (7). Since most IEl patients have defects in humoral and/or cellular components of immunity, they have susceptibility to viral and bacterial infections (5). Recent studies have shown that innate immune cells and specific immune pathways such as interferon (IFN) pathways have protective roles against certain viruses and a normal response to SARS-CoV-2 may depend on an intact innate immunity (8). Mutations in genes expressing IFN receptors (IFNAR1 and IFNAR2) and IFN regulatory factors (IRF7, IRF9, IFIH1) have been suggested as variants associated with severe COVID-19 disease $(8,9)$.

There are reports of increased frequency and severity of some IEls during the COVID-19 pandemic, such as common variable immune deficiency (CVID) (10). In contrast, a complete absence of B cells as seen in the cases of X-linked agammaglobulinemia (XLA) may have protective effects for COVID-19 infection, probably due to prevention of inflammation development (11). However, there are still concerns about the COVID-19 severity and clinical consequences in the IEI population. This study aimed to assess the effect of SARS-CoV-2 infection on patients with IEls, regarding the clinical course, complications, severity, and outcomes.

\section{li. Methods}

This retrospective study was conducted on patients from three referral immunodeficiency centers located in Mofid children's hospital and Masih Daneshvari hospital in Tehran and Namazi hospital in Shiraz provinces from March 2020 to May 2021.

All three participating centers in this study had access to national guidelines and necessary laboratory equipment for clinical and immunological evaluations of IEI patients. Moreover, the clinical diagnosis of the IEl patients was based on the diagnostic criteria of the European Society for immunodeficiencies (ESID)

Page 3/12 
working party (12).

The study was performed in two phases:

- The phase 1 study was performed in Mofid children's hospital and Masih Daneshvari hospital in Tehran during the early months of the COVID-19 epidemic, from March 2020 to mid-July, 2020. Inclusion criteria were; 1) Patients with an established diagnosis of IEls, 2) COVID-19 patients who tested positive for nucleic acid, 3) COVID-19 patients whose clinical stages were sorted based on currently accepted clinical stages. Patients who refused to cooperate were excluded. A questionnaire was surveyed by a phone interview to collect patient's demographic data, the clinical manifestation of COVID-19, probable transmission route, and measures taken against COVID-19.

- The phase 2 study was a retrospective cross-sectional study performed in three above-mentioned centers, and all IEls patients with COVID-19 infection from March 2020 to May 2021, were included. A questionnaire inquired multiple variables including demographic data such as age and sex of patients, IEl type and category, age at onset of immunodeficiency disease and age of its diagnosis, presence of comorbidities, past drug history (e.g. prophylactic antibiotics, immunoglobulin replacement therapy, current immunosuppressive therapy, etc.), clinical manifestations and laboratory data of COVID-19 infection, suspicion of COVID-19 infection or its confirmation by reverse transcription-polymerase chain reaction (RT-PCR) from nasopharyngeal samples, need for outpatient care or hospitalization or intensive care unit admission, types of treatment required, COVID-19 complications and outcomes.

All analyses were implemented using SPSS software (Version 26.0, Chicago, IL) and continuous quantitative variables were summarized with mean, standard deviation (SD), median, and interquartile range (IQR), whereas categorical and qualitative variables were recorded with frequencies and percentages. Statistical analysis was provided by using Mann-Whitney, Chi-square, and Fisher exact tests. P values less than 0.05 were considered statistically significant.

This study received approval from the Ethics Committee of the Shahid Beheshti University of Medical science (Approval code: IR.SBMU.RICH.REC.1400.025). Written informed consent was obtained from all patients and/or their parents.

\section{lii. Results}

\section{III.A. Demographic and clinical features of COVID-19 in patients with inborn errors of immunity - Phase 1}

At the beginning of COVID-19 pandemic, 93 IEls patients [with gender distribution of $60.2 \%$ male and $39.8 \%$ female] were evaluated by telephone follow-up (TFU) including 22 (23.6\%) CVID, 18 (19.35\%) combined immunodeficiency (CID), 11 (11.82\%) chronic granulomatous disease (CGD), 8 (8.6\%) hyper IgM syndrome (HIGM), 7 (7.52\%) Mendelian susceptibility to mycobacterial diseases (MSMD), 5 (5.37\%) cyclic neutropenia (CN), 4 (4.3\%) Wiskott-Aldrich syndrome (WAS), 4 (4.3\%) ataxia telangiectasia, 4 (4.3\%) IgA deficiency (IgAD), 3 (3.22\%) XLA, 3 (3.22\%) hyper IgE syndrome, and autoimmune lymphoproliferative syndrome (ALPS), familial Mediterranean fever (FMF), Chédiak-Higashi Syndrome (CHS), and leukocyte adhesion deficiency (LAD) each in $1(1.07 \%)$ patient.

All patients implemented basic measures against COVID-19, including hand wash, avoiding busy places, keeping distance, and using sanitary products to surface. Most patients $(n=74,79.5 \%)$ wore facial mask.

Among 93 PID patients, 16 patients (17.2\%) including 3 HIGM, 3 CVID, 3 CID, 1 IgAD, 1 CN, 1 WAS, and 1 XLA patients developed COVID-19 symptoms. 3 out of 16 patients were PCR-confirmed cases and the others were diagnosed clinically. The most commonly reported signs and symptoms were fever (10 cases), diarrhea ( 6 cases), cough (3 cases), otalgia, sore throat, and cervical lymphadenopathy (3 cases collectively), respiratory distress ( 2 cases), and rash (1 case). Four patients (25\%) required hospitalization, though they were in stable condition in the general ward and only received supportive care. There was no pediatric invasive care unit (PICU) admission. No death was reported in this phase.

\section{III.B. Demographic and clinical features of COVID-19 in patients with inborn errors of immunity - Phase 2}

\section{A. Demographic and clinical features of COVID-19 in patients with inborn errors of immunity}

A total of 33 patients, $57.6 \%$ male $(n=19)$ and $42.4 \%$ female $(n=14)$, with an established diagnosis of IEI were enrolled in this study. Based on the latest IUIS phenotypical classification (13), most of the patients were categorized into combined immunodeficiency (CID) ( $n=14,42.4 \%)$ and predominantly antibody deficiencies $(n=11,33.3 \%$ ), followed by less common categories of congenital defects of phagocyte number and function ( $n=3,9.1 \%)$, severe combined immunodeficiency (SCID) $(n=2,6.1 \%)$, diseases of immune dysregulation $(n=2,6.1 \%)$, and defects in intrinsic and innate immunity $(n=1,3.0 \%)($ Table 1 and Figure 1). According to geographical distribution of IEls proposed by Iranian Primary Immunodeficiency Registry (IPIDR) (ref), more than half of the patients were referred from high PID incidence (>50/million) ( $n=19,57.6 \%)$, and then from moderate PID incidence (10-50/million) ( $n=12,36.4 \%)$, and low PID incidence ( $<10 /$ million) $(n=2,6.1 \%)$. 
Table 1

Summary of demographic data, clinical manifestations and outcomes

\begin{tabular}{|c|c|c|c|c|c|c|c|c|c|}
\hline $\begin{array}{l}\text { Pt } \\
\text { ID }\end{array}$ & $\begin{array}{l}\text { City of } \\
\text { residence }\end{array}$ & Sex & $\begin{array}{l}\text { Age of } \\
\text { admission } \\
\text { (Months) }\end{array}$ & IEl type & Comorbidities & Drug history & $\begin{array}{l}\text { COVID19 } \\
\text { Manifestations }\end{array}$ & $\begin{array}{l}\text { COVID-19 } \\
\text { PCR } \\
\text { (\#attempt) }\end{array}$ & $\begin{array}{l}\text { In-hospital } \\
\text { Medications }\end{array}$ \\
\hline 1 & Varamin & M & 163 & Bruton & None & $\begin{array}{l}\text { Prophylactic } \\
A B \text {, IVlg }\end{array}$ & $\begin{array}{l}\text { Fever, Dry } \\
\text { cough, runny } \\
\text { nose/sneezing, } \\
\text { Dyspnea }\end{array}$ & $\begin{array}{l}\text { Positive } \\
\text { (1st) }\end{array}$ & $\begin{array}{l}\text { Clindamycin } \\
\text { Amikacin, IV }\end{array}$ \\
\hline 2 & Garme & M & 107 & Hyper IgM & None & IVIg & $\begin{array}{l}\text { Fever, Wet } \\
\text { cough, GI } \\
\text { symptoms, } \\
\text { Convulsion }\end{array}$ & $\begin{array}{l}\text { Positive } \\
\text { (1st) }\end{array}$ & $\begin{array}{l}\text { Meropenem, } \\
\text { Vancomycin } \\
\text { Metronidazo } \\
\text { IVIg }\end{array}$ \\
\hline 3 & Ghiamdasht & M & 112 & Ataxia telangiectasia & None & IVIg & $\begin{array}{l}\text { Fever, runny } \\
\text { nose/sneezing, } \\
\text { Gl symptoms, } \\
\text { Myalgia }\end{array}$ & $\begin{array}{l}\text { Positive } \\
\text { (1st) }\end{array}$ & $\begin{array}{l}\text { Meropenem, } \\
\text { Vancomycin } \\
\text { IVlg }\end{array}$ \\
\hline 4 & Shahriar & $\mathrm{F}$ & 48 & BCGosis & $\begin{array}{l}\text { Fanconi syndrome, } \\
\text { GI disorder }\end{array}$ & $\begin{array}{l}\text { IVIg, IS, } \\
\text { Favipiravir }\end{array}$ & $\begin{array}{l}\text { Fever, Dry } \\
\text { cough, } \\
\text { Tachypnea, } \\
\text { Hypotension, } \\
\text { Retraction, } \\
\text { Respiratory } \\
\text { distress, GI } \\
\text { symptoms, } \\
\text { Myalgia }\end{array}$ & $\begin{array}{l}\text { Positive } \\
\text { (1st) }\end{array}$ & $\begin{array}{l}\text { Meropenem, } \\
\text { Cotrimoxazc } \\
\text { Corticosteroi }\end{array}$ \\
\hline 5 & Varamin & M & 1.5 & CID & $\begin{array}{l}\text { Severe pneumonia, } \\
\text { GI disorder, after } \\
\text { COVID- } 19 \text { seizure } \\
\text { and pericardial } \\
\text { effusion }\end{array}$ & $\begin{array}{l}\text { Prophylactic } \\
\text { AB, IVlg }\end{array}$ & $\begin{array}{l}\text { Fever, Dry } \\
\text { cough, } \\
\text { Dyspnea, GI } \\
\text { symptoms }\end{array}$ & $\begin{array}{l}\text { Positive } \\
\text { (1st) }\end{array}$ & $\begin{array}{l}\text { Meropenem, } \\
\text { Vancomycin } \\
\text { Amikacin, } \\
\text { Nystatin, } \\
\text { Fluconazole } \\
\text { Corticosteroi }\end{array}$ \\
\hline 6 & Ghazvin & M & 6.5 & SCID & $\begin{array}{l}\text { Atopy, } \\
\text { Organomegaly }\end{array}$ & $\begin{array}{l}\text { IVIg, } \\
\text { Ganciclovir }\end{array}$ & $\begin{array}{l}\text { Fever, Dyspnea, } \\
\text { Tachypnea, } \\
\text { Hypotension, } \\
\text { Retraction, } \\
\text { Respiratory } \\
\text { distress }\end{array}$ & $\begin{array}{l}\text { Positive } \\
\text { (3rd) }\end{array}$ & $\begin{array}{l}\text { Vancomycin } \\
\text { Meropenem, } \\
\text { CotrimoxazC } \\
\text { Rifampin, IN } \\
\text { Ethambutol, } \\
\text { Clotrimazole } \\
\text { Corticosteroi } \\
\text { IVIg }\end{array}$ \\
\hline 7 & Qom & $\mathrm{F}$ & 179.7 & CVID & Appendicitis & $\begin{array}{l}\text { Prophylactic } \\
\text { AB, IVlg, } \\
\text { Acyclovir }\end{array}$ & $\begin{array}{l}\text { Fever, GI } \\
\text { symptoms, } \\
\text { Myalgia }\end{array}$ & $\begin{array}{l}\text { Positive } \\
\text { (1st) }\end{array}$ & $\begin{array}{l}\text { Cotrimoxazc } \\
\text { Clindamycin } \\
\text { Fluconazole, } \\
\text { IVlg }\end{array}$ \\
\hline 8 & Kordestan & M & 13 & CID & Allergic colitis & $\begin{array}{l}\text { Prophylactic } \\
\text { AB, IVIg, IS, } \\
\text { Chloroquine }\end{array}$ & GI symptoms & $\begin{array}{l}\text { Positive } \\
\text { (1st) }\end{array}$ & $\begin{array}{l}\text { Ceftriaxone, } \\
\text { Corticosteroi } \\
\text { IVlg }\end{array}$ \\
\hline 9 & Babol & $\mathrm{F}$ & 32.2 & Gaucher, Neutropenia & Organomegaly & $\begin{array}{l}\text { IVIg, } \\
\text { Chloroquine }\end{array}$ & $\begin{array}{l}\text { Fever, Dry } \\
\text { cough, Gl } \\
\text { symptoms }\end{array}$ & Negative & $\begin{array}{l}\text { Ceftriaxone, } \\
\text { Vancomycin }\end{array}$ \\
\hline 10 & Jajrood & $\mathrm{F}$ & 231.7 & $\begin{array}{l}\text { SLE, Scleroderma, } \\
\text { MCTD }\end{array}$ & $\begin{array}{l}\text { SLE, Scleroderma, } \\
\text { RTA, seizure, lung } \\
\text { disease }\end{array}$ & $\begin{array}{l}\text { IVIg, } \\
\text { Remdesivir }\end{array}$ & $\begin{array}{l}\text { Gl symptoms, } \\
\text { Convulsion, } \\
\text { LOC }\end{array}$ & $\begin{array}{l}\text { Positive } \\
\text { (1st) }\end{array}$ & $\begin{array}{l}\text { Meropenem, } \\
\text { Vancomycin } \\
\text { Corticosteroi } \\
\text { IVIg }\end{array}$ \\
\hline 11 & Qom & $\mathrm{F}$ & 18 & $\begin{array}{l}\text { Early onset IBD/IL10R } \\
\text { defect }\end{array}$ & $\begin{array}{l}\text { Early onset IBD, } \\
\text { Food allergy }\end{array}$ & - & - & $\begin{array}{l}\text { Positive } \\
\text { (2nd ) }\end{array}$ & $\begin{array}{l}\text { Clindamycin } \\
\text { Amikacin, } \\
\text { Meropenem, } \\
\text { Vancomycin } \\
\text { Azithromycir } \\
\text { Corticosteroi }\end{array}$ \\
\hline 12 & Meygoon & M & 33.2 & CID & None & $\begin{array}{l}\text { Prophylactic } \\
\text { AB, IVlg, IS }\end{array}$ & $\begin{array}{l}\text { Fever, } \\
\text { Hypotension, } \\
\text { Gl symptoms, } \\
\text { LOC }\end{array}$ & Negative & $\begin{array}{l}\text { Meropenem, } \\
\text { Vancomycin } \\
\text { Fluconazole }\end{array}$ \\
\hline 13 & Eslamshahr & M & 7.7 & Ataxia telangiectasia & $\begin{array}{l}\text { Hodgkin } \\
\text { lymphoma, } \\
\text { Pericardial } \\
\text { effusion }\end{array}$ & $\begin{array}{l}\text { IVIg, } \\
\text { Ganciclovir }\end{array}$ & $\begin{array}{l}\text { Dyspnea, } \\
\text { Tachypnea, } \\
\text { Hypotension, } \\
\text { Retraction, } \\
\text { Respiratory } \\
\text { distress }\end{array}$ & Negative & $\begin{array}{l}\text { Meropenem, } \\
\text { Vancomycin } \\
\text { Voriconazolı } \\
\text { Cotrimoxazc } \\
\text { IVlg, } \\
\text { Corticosteroi }\end{array}$ \\
\hline 14 & Shahriar & $\mathrm{F}$ & 81.2 & CVID & None & IVIg & $\begin{array}{l}\text { Fever, Dry } \\
\text { cough, GI } \\
\text { symptoms }\end{array}$ & $\begin{array}{l}\text { Positive } \\
\text { (1st) }\end{array}$ & $\begin{array}{l}\text { Ceftriaxone, } \\
\text { Vancomycin }\end{array}$ \\
\hline
\end{tabular}




\begin{tabular}{|c|c|c|c|c|c|c|c|c|c|}
\hline $\begin{array}{l}\mathrm{Pt} \\
\text { ID }\end{array}$ & $\begin{array}{l}\text { City of } \\
\text { residence }\end{array}$ & Sex & $\begin{array}{l}\text { Age of } \\
\text { admission } \\
\text { (Months) }\end{array}$ & IEl type & Comorbidities & Drug history & $\begin{array}{l}\text { COVID19 } \\
\text { Manifestations }\end{array}$ & $\begin{array}{l}\text { COVID-19 } \\
\text { PCR } \\
\text { (\#attempt) }\end{array}$ & $\begin{array}{l}\text { In-hospital } \\
\text { Medications }\end{array}$ \\
\hline 15 & Shiraz & $\mathrm{F}$ & 31 & STIM1 deficiency & $\begin{array}{l}\text { Vasculitis, } \\
\text { Nephrotic } \\
\text { syndrome, } \\
\text { Myopathy, } \\
\text { Autoimmune } \\
\text { anemia and } \\
\text { thrombocytopenia }\end{array}$ & IVIg, IS & $\begin{array}{l}\text { Fever, Dry } \\
\text { cough, } \\
\text { Dyspnea, } \\
\text { Cyanosis, } \\
\text { Tachypnea, } \\
\text { Retraction, } \\
\text { Respiratory } \\
\text { distress }\end{array}$ & $\begin{array}{l}\text { Positive } \\
\text { (2nd) }\end{array}$ & $\begin{array}{l}\text { Vancomycin } \\
\text { Tazocin, } \\
\text { Meropenem, } \\
\text { Corticosteroi } \\
\text { IVlg }\end{array}$ \\
\hline 16 & Darab & $\mathrm{F}$ & 6 & SCID & $\begin{array}{l}\text { Atopy, Otitis } \\
\text { media, } \\
\text { Organomegaly, } \\
\text { Lymphadenopathy, } \\
\text { VSD, DCM, } \\
\text { Convulsion }\end{array}$ & $\begin{array}{l}\text { Prophylactic } \\
\text { AB, IVlg, IS, } \\
\text { Acyclovir }\end{array}$ & $\begin{array}{l}\text { Fever, Dry } \\
\text { cough, } \\
\text { Dyspnea, } \\
\text { Cyanosis, } \\
\text { Tachypnea, } \\
\text { Retraction, } \\
\text { Convulsion, } \\
\text { LOC }\end{array}$ & $\begin{array}{l}\text { Positive } \\
\text { (1st) }\end{array}$ & $\begin{array}{l}\text { Clindamycin } \\
\text { Meropenem, } \\
\text { fluconazole, } \\
\text { Corticosteroi } \\
\text { IVlg }\end{array}$ \\
\hline 17 & Shiraz & $M$ & 217 & ICF syndrome & $\begin{array}{l}\text { Organomegaly, } \\
\text { Cirrhosis }\end{array}$ & $\begin{array}{l}\text { IVIg, } \\
\text { Remdesivir }\end{array}$ & Fever & $\begin{array}{l}\text { Positive } \\
\text { (1st) }\end{array}$ & $\begin{array}{l}\text { Vancomycin } \\
\text { Tazocin, } \\
\text { Corticosteroi } \\
\text { IVlg }\end{array}$ \\
\hline 18 & Shiraz & M & 324 & Bruton & Bronchiectasis & IVlg & $\begin{array}{l}\text { Fever, Dry } \\
\text { cough, } \\
\text { Myalgia, } \\
\text { Anosmia }\end{array}$ & $\begin{array}{l}\text { Positive } \\
\text { (1st) }\end{array}$ & $\begin{array}{l}\text { Azithromycir } \\
\text { IVlg }\end{array}$ \\
\hline 19 & Shiraz & $M$ & 264 & Bruton & $\begin{array}{l}\text { Hip Dysplasia, } \\
\text { Poliomyelitis }\end{array}$ & $\begin{array}{l}\text { IVIg, } \\
\text { Remdesivir }\end{array}$ & $\begin{array}{l}\text { Fever, Dry } \\
\text { cough, Runny } \\
\text { nose/Sneezing, } \\
\text { Myalgia }\end{array}$ & Negative & $\begin{array}{l}\text { Vancomycin } \\
\text { Tazocin, } \\
\text { Corticosteroi } \\
\text { IVlg }\end{array}$ \\
\hline 20 & Tehran & $\mathrm{F}$ & 62.5 & CID & $\begin{array}{l}\text { Organomegaly, } \\
\text { Rheumatologic } \\
\text { disorder, } \\
\text { Autoimmunity }\end{array}$ & $\begin{array}{l}\text { Prophylactic } \\
\text { AB, IVIg, IS, } \\
\text { Chloroquine, } \\
\text { Valganciclovir, } \\
\text { ASA }\end{array}$ & $\begin{array}{l}\text { Fever, Dry } \\
\text { cough, Runny } \\
\text { nose/Sneezing, } \\
\text { Dyspnea, } \\
\text { Tachypnea, } \\
\text { Hypotension, } \\
\text { Retraction, GI } \\
\text { symptoms, } \\
\text { Myalgia }\end{array}$ & Negative & $\begin{array}{l}\text { AB, } \\
\text { Fluconazole, } \\
\text { Corticosteroi } \\
\text { IVlg, } \\
\text { Convalescen } \\
\text { plasma }\end{array}$ \\
\hline 21 & Tehran & $M$ & 86.5 & CID & Atopy & $\begin{array}{l}\text { Prophylactic } \\
A B \text {, IS }\end{array}$ & $\begin{array}{l}\text { Fever, Dry } \\
\text { cough, Myalgia }\end{array}$ & Negative & $A B, I V I g$ \\
\hline 22 & Khoramabad & M & 103 & CGD & $\begin{array}{l}\text { Liver, ENT, Lung } \\
\text { disorder, } \\
\text { Bronchiectasis, } \\
\text { Atopy }\end{array}$ & $\begin{array}{l}\text { Prophylactic } \\
\text { AB, IS }\end{array}$ & $\begin{array}{l}\text { Fever, Dry } \\
\text { cough, Runny } \\
\text { nose/Sneezing, } \\
\text { Dyspnea, } \\
\text { Cyanosis, } \\
\text { Tachypnea, } \\
\text { Hypotension, } \\
\text { Retraction, } \\
\text { Myalgia }\end{array}$ & Negative & $\begin{array}{l}\text { AB, } \\
\text { Fluconazole, } \\
\text { Corticosteroi } \\
\text { Monoclonal } \\
\text { antibody }\end{array}$ \\
\hline 23 & Varamin & $M$ & 6 & MSMD & $\begin{array}{l}\text { Lung disorder, } \\
\text { Bronchiectasis, } \\
\text { ENT disorder, } \\
\text { Organomegaly }\end{array}$ & $\begin{array}{l}\text { Prophylactic } \\
\text { AB, IVIg }\end{array}$ & $\begin{array}{l}\text { Fever, Dry } \\
\text { cough, Runny } \\
\text { nose/Sneezing, } \\
\text { Cyanosis, } \\
\text { Dyspnea, } \\
\text { Tachypnea, } \\
\text { Hypotension, } \\
\text { Myalgia }\end{array}$ & Negative & $\begin{array}{l}\text { AB, } \\
\text { Amphotericiı } \\
\text { IVlg }\end{array}$ \\
\hline 24 & Tehran & $M$ & 6.8 & CID & $\begin{array}{l}\text { Bronchiectasis, } \\
\text { Atopy }\end{array}$ & $\begin{array}{l}\text { Prophylactic } \\
\text { AB, IVIg, } \\
\text { Chloroquine }\end{array}$ & $\begin{array}{l}\text { Fever, Dry } \\
\text { cough }\end{array}$ & $\mathrm{NP}$ & $A B, I V I g$ \\
\hline 25 & Tehran & $\mathrm{F}$ & 224.7 & CVID & $\begin{array}{l}\text { Atopy, } \\
\text { Autoimmunity }\end{array}$ & $\begin{array}{l}\text { Prophylactic } \\
\text { AB, IVIg, IS }\end{array}$ & $\begin{array}{l}\text { Fever, Dry } \\
\text { cough, Runny } \\
\text { nose/Sneezing, } \\
\text { Dyspnea, } \\
\text { Cyanosis, } \\
\text { Tachypnea, } \\
\text { Retraction, } \\
\text { Myalgia }\end{array}$ & Negative & $\begin{array}{l}\text { AB, } \\
\text { Corticosteroi } \\
\text { IVlg }\end{array}$ \\
\hline 26 & Lahijan & Male & 51.7 & CID & ENT disorder & $\begin{array}{l}\text { Prophylactic } \\
\text { AB, IVIg, IS }\end{array}$ & $\begin{array}{l}\text { Fever, Dry } \\
\text { cough, } \\
\text { Tachypnea, }\end{array}$ & NP & $\begin{array}{l}\text { Voriconazolt } \\
\mathrm{AB} \text {, IVlg }\end{array}$ \\
\hline
\end{tabular}




\begin{tabular}{|c|c|c|c|c|c|c|c|c|c|}
\hline $\begin{array}{l}\text { Pt } \\
\text { ID }\end{array}$ & $\begin{array}{l}\text { City of } \\
\text { residence }\end{array}$ & Sex & $\begin{array}{l}\text { Age of } \\
\text { admission } \\
\text { (Months) }\end{array}$ & IEl type & Comorbidities & Drug history & $\begin{array}{l}\text { COVID19 } \\
\text { Manifestations }\end{array}$ & $\begin{array}{l}\text { COVID-19 } \\
\text { PCR } \\
\text { (\#attempt) }\end{array}$ & $\begin{array}{l}\text { In-hospital } \\
\text { Medications }\end{array}$ \\
\hline 27 & Maragheh & $\mathrm{F}$ & 16.4 & CID & $\begin{array}{l}\text { Neurologic } \\
\text { disorder }\end{array}$ & $\begin{array}{l}\text { Prophylactic } \\
\text { AB, IVlg, IS, } \\
\text { Chloroquine, } \\
\text { Naproxen }\end{array}$ & $\begin{array}{l}\text { Fever, Dry } \\
\text { cough, Runny } \\
\text { nose/Sneezing, } \\
\text { Dyspnea, } \\
\text { Tachypnea, } \\
\text { Hypotension, } \\
\text { Retraction, GI } \\
\text { symptoms, } \\
\text { Myalgia }\end{array}$ & Negative & $\begin{array}{l}\text { AB, } \\
\text { Corticosteroi } \\
\text { IVlg, } \\
\text { Fluconazole }\end{array}$ \\
\hline 28 & Tehran & $\mathrm{F}$ & 84.3 & Neutropenia & None & $\begin{array}{l}\text { Prophylactic } \\
\text { AB, Acyclovir }\end{array}$ & $\begin{array}{l}\text { Fever, Dry } \\
\text { cough, } \\
\text { Dyspnea, } \\
\text { Cyanosis, } \\
\text { Tachypnea, } \\
\text { Hypotension, } \\
\text { Retraction, GI } \\
\text { symptoms, } \\
\text { Myalgia }\end{array}$ & NP & $\begin{array}{l}\text { AB, } \\
\text { Fluconazole }\end{array}$ \\
\hline 29 & Sari & M & 139.7 & CVID & $\begin{array}{l}\text { ENT disorder, Lung } \\
\text { disorder, } \\
\text { Bronchiectasis, } \\
\text { Atopy, } \\
\text { Autoimmunity }\end{array}$ & $\begin{array}{l}\text { Prophylactic } \\
\text { AB, IVIg, } \\
\text { Chloroquine }\end{array}$ & $\begin{array}{l}\text { Fever, Dry } \\
\text { cough, runny } \\
\text { nose/sneezing, } \\
\text { Dyspnea, } \\
\text { Cyanosis, } \\
\text { Tachypnea, } \\
\text { Retraction, } \\
\text { Myalgia }\end{array}$ & NP & $\begin{array}{l}\mathrm{AB}, \\
\text { Voriconazole } \\
\text { IVlg }\end{array}$ \\
\hline 30 & Ardebil & $\mathrm{F}$ & 19.3 & CID & $\begin{array}{l}\text { Atopy, Heart } \\
\text { disease, } \\
\text { Hydrocephalus, } \\
\text { Renal disorder }\end{array}$ & $\begin{array}{l}\text { Prophylactic } \\
\text { AB, IVIg, IS, } \\
\text { Acyclovir }\end{array}$ & $\begin{array}{l}\text { Fever, Dry } \\
\text { cough, Runny } \\
\text { nose/Sneezing, } \\
\text { Dyspnea, } \\
\text { Cyanosis, } \\
\text { Tachypnea, } \\
\text { Retraction, GI } \\
\text { symptoms, } \\
\text { Myalgia, } \\
\text { Convulsion }\end{array}$ & $\begin{array}{l}\text { Positive } \\
\text { (1st) }\end{array}$ & $\begin{array}{l}\text { AB, } \\
\text { Voriconazole } \\
\text { Corticosteroi } \\
\text { IVlg }\end{array}$ \\
\hline 31 & Esfahan & $\mathrm{F}$ & 250 & $\begin{array}{l}\text { Autosomal recessive } \\
\text { Agammaglobulinemia }\end{array}$ & $\begin{array}{l}\text { ENT disorder, Lung } \\
\text { disorder, } \\
\text { Bronchiectasis, } \\
\text { Neurologic } \\
\text { disorder }\end{array}$ & $\begin{array}{l}\text { IVIg, IS, } \\
\text { Chloroquine, } \\
\text { Remdesivir, } \\
\text { Naproxen }\end{array}$ & $\begin{array}{l}\text { Fever, Dry } \\
\text { cough, Runny } \\
\text { nose/Sneezing, } \\
\text { Dyspnea, } \\
\text { Myalgia }\end{array}$ & $\begin{array}{l}\text { Positive } \\
\text { (1st) }\end{array}$ & $\begin{array}{l}\text { AB, } \\
\text { Caspofungir } \\
\text { Corticosteroi } \\
\text { IVlg }\end{array}$ \\
\hline 32 & Golpayegan & M & 316 & Bruton & $\begin{array}{l}\text { Lung disorder, } \\
\text { Bronchiectasis }\end{array}$ & $\begin{array}{l}\text { IVIg, IS, } \\
\text { Remdesivir, } \\
\text { Naproxen }\end{array}$ & $\begin{array}{l}\text { Fever, Dry } \\
\text { cough, runny } \\
\text { nose/sneezing, } \\
\text { Dyspnea, } \\
\text { Myalgia }\end{array}$ & $\begin{array}{l}\text { Positive } \\
\text { (1st) }\end{array}$ & $\begin{array}{l}\text { AB, } \\
\text { Corticosteroi } \\
\text { IVlg }\end{array}$ \\
\hline 33 & Tehran & M & 516.7 & CVID & $\begin{array}{l}\text { GI disorder, Heart } \\
\text { disease, ENT } \\
\text { disorder, Lung } \\
\text { disorder, } \\
\text { Bronchiectasis, } \\
\text { Organomegaly }\end{array}$ & $\begin{array}{l}\text { IVlg, IS, } \\
\text { Chloroquine, } \\
\text { Remdesivir, } \\
\text { Favipiravir, } \\
\text { Naproxen }\end{array}$ & $\begin{array}{l}\text { Fever, Dry } \\
\text { cough, Runny } \\
\text { nose/Sneezing, } \\
\text { Dyspnea, } \\
\text { Cyanosis, } \\
\text { Tachypnea, } \\
\text { Hypotension, } \\
\text { Retraction, GI } \\
\text { symptoms, } \\
\text { Myalgia }\end{array}$ & $\begin{array}{l}\text { Positive } \\
\text { (1st) }\end{array}$ & $\begin{array}{l}\text { AB, } \\
\text { Corticosteroi } \\
\text { IVIg }\end{array}$ \\
\hline
\end{tabular}

IEl; inborn errors of immunity, COVID-19; Coronavirus disease 2019, ICU; Intensive Care Unit, IVIg; Intravenous immunoglobulin, Gl; Gastrointestinal, AB; Antibic Ear nose throat, CID; Combined immunodeficiency, CVID; Common variable immunodeficiency, SCID; Severe combined immunodeficiency, IBD; Inflammatory $t$ Mendelian susceptibility to mycobacterial disease, ICF syndrome; Immunodeficiency with centromeric instability and facial anomalies, STIM1; Stromal intera granulomatous disease, NP; not performed, DIC; disseminated intravascular coagulation

The median (IQR) age of study population was 81 (28-198) months. Pre-existing comorbidities included atopy ( $n=9,27.3 \%)$, lung disorders ( $n=9,27.3 \%)$, neurologic disorders $(n=8,24.2 \%)$, bronchiectasis $(n=8,24.2 \%)$, organomegaly $(n=7,21.2 \%)$, ear-nose-throat disorders $(n=7,21.2 \%)$, gastrointestinal disorders $(n=6,18.2 \%)$, autoimmunity $(n=5,15.2 \%)$, cardiovascular disorders $(n=5,15.2 \%)$, rheumatologic disorders $(n=4,12.1 \%)$, renal disorders $(n=4,12.1 \%)$, liver disorders $(n=2,6.1 \%)$, and malignancy $(n=1,3.0 \%)$. Among these comorbidities, organomegaly $(p=0.030)$ and renal disorders $(p=0.033)$ were significantly associated with the development of respiratory insufficiency.

Before COVID-infection, all IEl patients were stable on the standard of care treatment. Twenty-nine of 33 IEl patients (87.9\%) received monthly intravenous immunoglobulin (IVIG) substitution as standard therapy. Seventeen patients (51.5\%) were on prophylactic antibiotic therapy and fifteen patients (45.5\%) received immunosuppressive agents. 
The most common chief complaints at the presentation were fever $(n=29,87.9 \%)$, cough (dry: $23(69.7 \%)$, wet: $1(3.0 \%)$ ), dyspnea ( $n=17,51.5 \%)$, myalgia $(n=17,51.5 \%)$, gastrointestinal symptoms $(n=15,45.5 \%)$, runny nose/sneezing $(n=15,45.5 \%)$, tachypnea $(n=15,45.5 \%)$, intercostal retraction $(n=13,39.4 \%)$, hypotension $(n=10,30.3 \%)$, cyanosis $(n=9,27.3 \%)$, seizure $(n=4,12.1 \%)$, and loss of consciousness $(n=3,9.1 \%)$. In addition, 26 patients were hypoxic with inroom $\mathrm{O}_{2}$ saturation of less than $90 \%(n=16,48.5 \%)$, or $90-95 \%(n=10,30.3 \%)$.

Patients with cyanosis $(p=0.047)$, tachypnea $(p=0.003)$, intercostal retraction $(p=0.003)$, and seizure $(p=0.033)$ at presentation were significantly more likely to progress respiratory insufficiency. In addition, tachypnea, intercostal retraction, hypotension, and hypoxia were significantly correlated with PICU admission $(p=0.022, p=0.019, p=0.007$, and $p=0.039)$ and death outcome $(p=0.027, p=0.036, p=0.005$, and $p=0.027$, respectively.

All except one patient were hospitalized. One patient with X-linked Agammaglobulinemia (XLA) had mild disease and was treated as outpatient. During hospitalization, patients received antibiotics for potential bacterial co-infection or superinfections $(n=33,100 \%)$, hydroxychloroquine/chloroquine ( $n=8,24.2 \%$ ), antivirals ( $n=14,42.4 \%)$, non-steroidal anti-inflammatory drugs (NSAIDs) ( $n=5,15.2 \%)$, antifungals ( $n=15,45.5 \%)$, steroids ( $n=20,60.6 \%)$, monoclonal antibody $(n=1,3.0 \%)$, and convalescent plasma $(n=1,3.0 \%)$. Twenty-four patients $(72.7 \%)$ continued to receive IVIG during hospitalization.

Almost half of patients $(n=17,51.5 \%)$ required PICU admission due to respiratory distress $(n=12)$, septic shock $(n=3)$, bradycardia $(n=1)$, and loss of consciousness $(n=1)$. Progression to respiratory insufficiency occurred in 15 patients $(45.5 \%)$.

At the end of the survey, thirteen patients (39.4\%) died following cardiorespiratory arrest $(n=4)$, septic shock ( $n=3)$, disseminated intravascular coagulation $(n=3)$, pulmonary hemorrhage $(n=1)$, acute respiratory distress syndrome $(n=1)$, and hemophagocytic lymphohistiocytosis $(n=1)$. Of note, the most lethal COVID-19 infection among IEl entities was observed in patients with CID ( 6 of $13,46.2 \%)$, followed by SCID ( 2 of $13,15.4 \%)$, PADs ( 2 of $13,15.4 \%)$, congenital defects of phagocytes ( 2 of 13,15.4\%), and diseases of immune dysregulation ( 1 of $13,7.7 \%$ ). However, the exact course of COVID-19 related deaths in these IEI patients is unknown. $69.2 \%$ (9 out of 13) of patients with IEI who succumbed to SARS-CoV-2 infection had pre-existing co-morbidities.

\section{B. Laboratory and Imaging Findings:}

SARS-CoV-2 infection was determined by RT-PCR $(n=19,57.6 \%)$ or computed tomography (CT) scan pattern $(n=26,78.8 \%)$ or chest x-ray $(C X R)$ pattern $(n=21$, 63.6\%). The first PCR test was positive in 16 (55.2\%) and negative in $13(44.8 \%)$ out of 29 evaluated patients. Three negative PCR tests turned positive within a median of $6(3-10)$ days after the first result.

A summary of laboratory results is presented in Table 2 . The complete blood count was consistent with leukocytosis ( $n=13,40.6 \%)$, leukopenia ( $n=12,37.5 \%)$, neutrophilia $(n=10,32.3 \%)$, lymphocytopenia $(n=14,45.2 \%)$, and thrombocytopenia $(n=11,34.4 \%)$. High inflammatory markers including ESR and CRP were high in $20(62.5 \%)$ and $23(71.9 \%)$ patients, respectively. 
Table 2

- Comparative analysis between survived and deceased patients

\begin{tabular}{|c|c|c|c|c|}
\hline Parameter & Total & Alive & Deceased & P-value \\
\hline White blood cell $\times 10^{3}$ (cell/uL), median (IQR) & $8.8(3.8-15.0)$ & $9.0(5.5-13.4)$ & $5.8(2.0-13.5)$ & 0.552 \\
\hline Absolute neutrophils count $\times 10^{3}$ (cells $\left./ \mu \mathrm{L}\right)$, median (IQR) & $4.1(1.9-10.8)$ & $4.1(2.2-10.8)$ & $4.4(1.0-10.5)$ & 0.685 \\
\hline Absolute lymphocytes count $\times 10^{3}$ (cells $/ \mu \mathrm{L}$ ), median (IQR) & $1.3(0.8-3.0)$ & $1.3(0.8-3.0)$ & $1.1(0.6-4.1)$ & 0.655 \\
\hline Hemoglobulin (g/dl), median (IQR) & $9.7(8.1-11.8)$ & $10.6(8.8-12.3)$ & $9.3(7.6-10.2)$ & 0.053 \\
\hline Platelets $\times 10^{3}$ (cells $\left./ \mu \mathrm{L}\right)$, median (IQR) & $247(96.25-335.75)$ & $279(106-336)$ & $203(80-332)$ & 0.527 \\
\hline Erythrocyte sedimentation rate (mm/hour), median (IQR) & $38.5(15.2-69.5)$ & $33.0(15.0-65.0)$ & $49.0(14.0-82.5)$ & 0.478 \\
\hline C-Reactive Protein (CRP) (mg/L), median (IQR) & $3.0(2.0-3.0)$ & $2.0(2.0-3.0)$ & $3.0(3.0-30.0)$ & 0.041 \\
\hline Lactic Acid Dehydrogenase (U/L), median (IQR) & $692(520-780)$ & $653(534-752.7)$ & $770(414-1108)$ & 0.183 \\
\hline Creatinine level (mg/dL), median (IQR) & $0.6(0.4-0.7)$ & $0.6(0.5-0.8)$ & $0.6(0.4-0.8)$ & 0.630 \\
\hline Urea (mmol/L), median (IQR) & $10.3(6.7-15.0)$ & $8.0(6.2-14.0)$ & $12.0(8.5-20.0)$ & 0.102 \\
\hline Serum sodium (mEq/L), median (IQR) & $135.0(130.0-138.0)$ & $135.0(132.0-138.0)$ & $131.0(126.5-139.0)$ & 0.239 \\
\hline Serum potassium (mEq/L), median (IQR) & $3.8(3.5-4.3)$ & $3.9(3.5-4.5)$ & $3.7(3.4-4.1)$ & 0.743 \\
\hline Serum calcium (mg/dL), median (IQR) & $8.4(8.0-8.9)$ & $8.5(7.9-8.9)$ & $8.4(8.0-9.1)$ & 0.936 \\
\hline Serum phosphorus (mmol/L), median (IQR) & $3.4(3.0-4.0)$ & $3.2(2.7-3.9)$ & $3.4(3.0-4.6)$ & 0.201 \\
\hline Serum magnesium (mmol/L), median (IQR) & $1.9(1.7-2.0)$ & $1.8(1.7-2.0)$ & $2.0(1.7-2.1)$ & 0.184 \\
\hline Venous Blood gases PH (U), median (IQR) & $7.35(7.20-7.40)$ & $7.40(7.35-7.43)$ & $7.20(7.15-7.28)$ & 0.003 \\
\hline Venous Blood gases $\mathrm{HCO}(\mathrm{mmol} / \mathrm{L})$, median (IQR) & $21.6(15-25.0)$ & $22.5(19.4-24.7)$ & $15(12.0-25.2)$ & 0.216 \\
\hline Venous Blood gases PCO2 (mmHg), median (IQR) & $44.5(29.0-54.7)$ & $41.5(32.2-49.5)$ & $49.4(25.2-77.5)$ & 0.643 \\
\hline Prothrombin time (seconds), median (IQR) & $14.0(12.0-18.6)$ & $12.7(12.0-15.0)$ & $16.0(13.3-22.0)$ & 0.045 \\
\hline Partial thromboplastin Time (seconds), median (IQR) & $35.0(29.6-47.5)$ & $35.0(27.7-39.5)$ & $35.0(30.1-60.0)$ & 0.509 \\
\hline International normalized ratio (INR), median (IQR) & $1.2(1.0-1.8)$ & $1.0(1.0-1.4)$ & $1.3(1.1-2.2)$ & 0.053 \\
\hline Creatine phosphokinase (U/L), median (IQR) & $54.5(44.7-117.0)$ & $53.5(45.5-115.0)$ & $74.0(36.5-130.7)$ & 0.895 \\
\hline AST (U/L), median (IQR) & $52.0(34.2-71.2)$ & $41.0(31.5-66.5)$ & $64.0(33.5-82.0)$ & 0.379 \\
\hline ALT (U/L), median (IQR) & $26.5(19.0-75.5)$ & $23.0(19.0-72.0)$ & $31.0(19.0-79.2)$ & 0.531 \\
\hline ALP (U/L), median (IQR) & $319.0(225.0-652.0)$ & $299.5(233.0-619.0)$ & $325.0(172.0-982.0)$ & 0.844 \\
\hline Total Bilirubin ( $\mu$ mol/L), median (IQR) & $1.0(0.6-1.2)$ & $0.9(0.7-1.0)$ & $1.1(0.6-6.2)$ & 0.235 \\
\hline Direct Bilirubin ( $\mu \mathrm{mol} / \mathrm{L})$, median (IQR) & $0.2(0.1-0.6)$ & $0.2(0.2-0.5)$ & $0.3(0.1-3.6)$ & 0.581 \\
\hline Troponin (ng/mL), median (IQR) & $0.01(0-0.002)$ & $0(0-0.01)$ & $0.01(0-111.7)$ & 0.049 \\
\hline Ferritin ( $\mu \mathrm{g} / \mathrm{L})$, median (IQR) & $413(95-1500)$ & $125(78-413)$ & $800(800-2037)$ & 0.020 \\
\hline Fibrinogen (g/L), median (IQR) & $200(100.2-236.7)$ & $197.0(88.7-203.5)$ & $231.5(103.7-336.5)$ & 0.143 \\
\hline D-Dimer (ng/mL), median (IQR) & $200(140-200)$ & $190(86.5-200)$ & $200(200-748)$ & 0.045 \\
\hline
\end{tabular}

Serum creatinine and BUN were high in 18 (56.3\%) and 6 (18.8\%) and based on the KDIGO criteria were in the stage 1 AKI range in three patients. Evidence of proteinuria $(n=11,35.5 \%)$ and/or hematuria $(n=9,29 \%)$ was observed in 13 patients $(41.9 \%)$. The most common electrolyte abnormalities included hyponatremia $(n=14,43.8 \%)$, hypokalemia $(n=5,15.6 \%)$, hypocalcemia $(n=17,54.8 \%)$, hypophosphatemia $(n=23,79.3 \%)$, and hypomagnesemia $(n=15,55.6 \%)$. Half of the patients with evaluated VBG (10 of 20) had normal acid-base state, while metabolic acidosis $(n=5,25 \%)$, respiratory acidosis ( $n=4,20 \%)$, and respiratory alkalosis $(n=1,5 \%)$ were also reported.

High serum levels of LDH (21 of 27, 77.8\%), PT (10 of 29, 34.5\%), PTT (9 of 29, 31\%), INR (13 of 29, $44.8 \%$ ), CPK (4 of $22,18.2 \%)$, AST (18 of $30,60 \%)$, ALT (10 of $30,33 \%)$, ALK-P ( 2 of $27,7.4 \%$ ), bilirubin (total: 6 of $18,33.3 \%$, direct: 5 of $18,27.8 \%$ ), troponin $(2$ of $13,15.4 \%)$, ferritin (13 of $19,68.4 \%)$, fibrinogen (1 of 20 , $5 \%)$, D-dimer (9 of $13,69.2 \%$ ) were also observed. 8 out of $11(72.7 \%)$ patients evaluated for serum vitamin D level were insufficient or deficient.

The mortality rate was correlated with high ferritin $(p=0.018)$, proteinuria $(p=0.056)$, leukopenia $(p=0.150)$, high troponin $(p=0.192)$, high $\mathrm{BUN}(p=0.194)$, hypocalcemia $(p=0.171)$, high PT $(p=0.064)$, high $\mathrm{D}$-dimer $(p=0.105)$, high Alk-P $(p=0.157)$, total bilirubin $(p=0.141)$ and death outcome. Furthermore, there was a significant correlation between hyponatremia $(p=0.011)$ and proteinuria $(p=0.013)$ and the need for PICU admission. Thrombocytopenia $(p=0.108)$,

Page 9/12 
hematuria $(p=0.113)$, and high serum levels of $\operatorname{LDH}(p=0.077)$, PT $(p=0.126)$, AST $(p=0.176)$, and total bilirubin $(p=0.141)$ were also correlate with the rate of PICU admission, although not statistically significant.

Positive PCR and COVID-19 evidence in CT scan were not associated with PICU admission ( $p=0.579$ and $p=0.654)$ or death outcome $(p=0.727$ and $p=0.361)$, respectively. However, evidence of COVID-19 in CXR was significantly associated with the need for PICU admission $(p=0.023)$ and death $(p=0.046)$.

\section{Iv. Discussion}

COVID-19 is a worldwide concern affecting millions of people globally and Iran is a hot zone area for this disease within the middle-east (14). Patients suffering from IEl are regarded as potentially at-risk cases during the current pandemic (15). However, the impact of SARS-CoV-2 on patients with primary immunodeficiency is not yet fully understood. Therefore, we retrospectively studied the clinical course and characteristics of COVID-19 in a number of Iranian patients with IEI. Up to this point, studies showed a higher incidence of COVID-19 in IEls considering their underlying immunological defects (16), whereas, some others pointed out a fewer risk of severity among these patients $(15,17)$. Similarly, in phase one of this study, we detected a prevalence of $16.3 \%$ for SARS-CoV-2 infection in patients with IEI through TFU which was higher than the estimated prevalence of COVID-19 among the Iranian population (18).

A large proportion of our patients in phase two had combined immunodeficiency and antibody deficiency being stable on the standard of care treatment. The pre-existing comorbidities as important determinants of poor outcome were present in $69.2 \%$ of the patients who succumbed to SARS-CoV-2 infection. Atopy and lung disorders were the main comorbidities detected in our patients. However, the presence of organomegaly and renal disorder was strongly correlated with the development of respiratory insufficiency, which may be considered as a severity index and a high risk of death. This relationship between chronic renal disorders and COVID-19 severity has been previously described in both the general populace $(19,20)$ and patients with IEls $(21)$.

Although a majority of clinical symptoms encompassing fever (87.9\%), dry cough (69.7\%), dyspnea (51.5\%), myalgia (51.5\%), and gastrointestinal symptoms $(45.5 \%)$ in our patients overlapped the general population, the frequency of atypical complaints including seizure (12.1\%) and loss of consciousness ( $9.1 \%)$ was more prominent than the general population and previous IEI study (22-24). Severe symptoms such as cyanosis, tachypnea, intercostal retraction, hypotension, and seizure were accompanied by a higher rate of respiratory insufficiency leading to PICU admission and death. Similar to Delavari S et al. study (16), the mortality rate (39.4\%) was considerably greater than the general population, of those, cases with CID were accounted for most. It should also be noted that our cases were predominantly diagnosed with CID.

The rate of false-negative first SARS-CoV-2 PCR test was $10.3 \%$ in our patients that is slightly higher than the results of a study conducted in Canada (25). Consistent with former publications that exhibited an association between increased inflammatory markers and worse prognosis in COVID-19 (26, 27), we found a remarkable positive correlation between D-Dimer, PT, CRP, proteinuria, ferritin, metabolic acidosis, and troponin level with mortality and poorer outcome. Similar to other studies in SARS-CoV-2 infection (28), hyponatremia (43.8\%) was one of the prevalent electrolyte imbalances leading to increased risk of PICU admission $(p=0.011)$.

We further tracked the radiographic evidence of COVID-19 in CXR and chest CT scans of the patients. Conforming to the reports of Colman J et al. (29), CXR patterns unlike chest CT scan and positive PCR test were significantly associated with ICU admission $(p=0.023)$ and death $(p=0.046)$. On the other hand, this skewing might be the result of higher demand for CXR in critically ill patients due to its portability and inability to reveal slight changes of lung fields compared to chest CT scans.

Interestingly, SARS-CoV-2 was the triggering factor that prompted to X-linked Hyper-lgM syndrome diagnosis in one of our patients who was a 7 months old boy who presented with pneumonia and cervical lymphadenopathy. He had lymphopenia, low CD4+ cell, CD8+ cell, natural killer (NK) cell counts along with low immunoglobulin level, as described previously in hospitalized COVID-19 patients (30). Three other novel cases with agammaglobulinemia (two X-linked and one autosomal recessive) represented prolonged disease and PCR-positivity for SARS-CoV-2 infection. Thus, a recommendation for prolonged treatment of patients with IEl, particularly agammaglobulinemia, might be of benefit.

In summary, we studied the course of COVID-19 in 33 patients with IEI. Our data revealed that pre-existing organomegaly and renal disorder along with severe SARS-CoV-2-related symptoms including cyanosis, tachypnea, intercostal retraction, hypotension, and seizure were the predisposing factors to PICU admission and mortality. Additionally, radiographic evidence of COVID-19 in CXR was remarkably associated with a worse prognosis in COVID-19. We also reported prolonged disease duration and PCR-positivity for SARS-CoV-2 infection in agammaglobulinemic patients and introduced SARS-CoV-2 as the triggering factor prompting IEl appearance. However, future studies should be conducted to provide further evidence for this conclusion.

\section{Declarations}

Funding: The authors received no specific funding for this research.

Conflicts of interest/Competing interests: The authors declare that they have no conflict of interest.

Availability of data and material: Not applicable.

Code availability: Not applicable.

Ethics approval: The present study was conducted according to the principles expressed in the Helsinki Declaration and the ethics committee of Pediatric Infections Research Center and Research Institute for Children's Health of Shahid Beheshti University of Medical Sciences (Approval code:

IR.SBMU.RICH.REC.1400.025). 
Consent to participate: The informed consent was obtained from all individual participants (their parents) included in the study.

Consent for publication: Informed consent for publication was obtained from the parents of the patient prior to being included in the study.

\section{References}

1. Song F, Shi N, Shan F, Zhang Z, Shen J, Lu H, et al. Emerging 2019 Novel Coronavirus (2019-nCoV) Pneumonia. Radiology. 2020;295(1):210-7.

2. Cevik M, Kuppalli K, Kindrachuk J, Peiris M. Virology, transmission, and pathogenesis of SARS-CoV-2. BMJ. 2020;371:m3862.

3. Worldometers. https://www.worldometers.info/coronavirus/worldwide-graphs. 2021.

4. Fadl N, Ali E, Salem TZ. COVID-19: Risk Factors Associated with Infectivity and Severity. Scand J Immunol. 2021;93(6):e13039.

5. Bousfiha A, Jeddane L, Picard C, Al-Herz W, Ailal F, Chatila T, et al. Human Inborn Errors of Immunity: 2019 Update of the IUIS Phenotypical Classification. J Clin Immunol. 2020;40(1):66-81.

6. Notarangelo LD, Bacchetta R, Casanova JL, Su HC. Human inborn errors of immunity: an expanding universe. Science immunology. 2020;5(49).

7. Abolhassani H, Azizi G, Sharifi L, Yazdani R, Mohsenzadegan M, Delavari S, et al. Global systematic review of primary immunodeficiency registries. Expert Review of Clinical Immunology. 2020;16(7):717-32.

8. Zhang Q, Bastard P, Liu Z, Le Pen J, Moncada-Velez M, Chen J, et al. Inborn errors of type I IFN immunity in patients with life-threatening COVID-19. Science. 2020;370(6515).

9. Bastard P, Rosen LB, Zhang Q, Michailidis E, Hoffmann HH, Zhang Y, et al. Autoantibodies against type I IFNs in patients with life-threatening COVID-19. Science. 2020;370(6515).

10. Weifenbach N, Jung A, Lötters S. COVID-19 infection in CVID patients: What we know so far. Immunity Inflammation Disease. 2021;9(3):632-4.

11. laboni A, Wong N, Betschel SD. A Patient with X-Linked Agammaglobulinemia and COVID-19 Infection Treated with Remdesivir and Convalescent Plasma. J Clin Immunol. 2021;41(5):923-5.

12. Mario Abinun MA, Cohen SB, Buckland M, Bustamante J, Cant A, Casanova J-L, Chapel H, Basile GS, de Vries E, Dokal I, Donadieu J, Durandy A, Edgar D, Ehl S, Espanol T, Etzioni A, Fischer A, Gaspar B, Gasteiger L, Gathmann B, Gatti R, Andrew Gennery, Sofia Grigoriadou, Bodo Grimbacher, Steven Holland, Gritta Janka, Maria Kanariou, Gerhard Kindle, Christoph Klein, Helen Lachmann, Desa Lilic, Nizar Mahlaoui, Ania Manson, Natalia Martinez, Isabelle Meyts, Nicolette Moes, Despina Moshous, Benedicte Neven, Hans Ochs, Capucine Picard, Isabella Quinti, Ellen Renner, Frederic Rieux-Laucat, Peter Robinson, Reinhard Seger, Markus Seidel, Annarosa Soresina, Dominique Stoppa-Lyonnet, Vojtech Thon, Adrian Thrasher, Frank van de Veerdonk, Joris van Montfrans, Anna Villa, Corry Weemaes, Klaus Warnatz, Beata Wolska, and Shen-Yin Zhang. https://esid.org/Working-Parties/Registry-WorkingParty/Diagnosis-criteria. 2019.

13. Bousfiha A, Jeddane L, Picard C, Al-Herz W, Ailal F, Chatila T, et al. Human Inborn Errors of Immunity: 2019 Update of the IUIS Phenotypical Classification. J Clin Immunol. 2020;40(1):66-81.

14. Organization WH. WHO Coronavirus (COVID-19) Dashboard 17 August 2021 [Available from: https://covid19.who.int/.

15. Quinti I, Mezzaroma I, Milito C. Clinical management of patients with primary immunodeficiencies during the COVID-19 pandemic. Expert Review of Clinical Immunology. 2021;17(2):163-8.

16. Delavari S, Abolhassani H, Abolnezhadian F, Babaha F, Iranparast S, Ahanchian H, et al. Impact of SARS-CoV-2 Pandemic on Patients with Primary Immunodeficiency. J Clin Immunol. 2021;41(2):345-55.

17. Marcus N, Frizinsky S, Hagin D, Ovadia A, Hanna S, Farkash M, et al. Minor Clinical Impact of COVID-19 Pandemic on Patients With Primary Immunodeficiency in Israel. Front Immunol. 2021;11:3505.

18. Khalagi K, Gharibzadeh S, Khalili D, Mansournia MA, Mirab Samiee S, Aghamohamadi S, et al. Prevalence of COVID-19 in Iran: results of the first survey of the Iranian COVID-19 Serological Surveillance programme. Clin Microbiol Infect. 2021.

19. Li X, Zhong X, Wang Y, Zeng X, Luo T, Liu Q. Clinical determinants of the severity of COVID-19: A systematic review and meta-analysis. PLOS ONE. 2021;16(5):e0250602.

20. Mohkam M, Mirzaee M, Abdollah Gorgi F, Rafiei Tabatabaei S, Karimi A, Armin S, et al. Renal Involvement in COVID-19 Among Iranian Children. Arch Pediatr Infect Dis. 2021;9(1):e106597.

21. Shields AM, Burns SO, Savic S, Richter AG. COVID-19 in patients with primary and secondary immunodeficiency: The United Kingdom experience. J Allergy Clin Immunol. 2021;147(3):870-5.e1.

22. Haghighi-Morad M, Alavi Darazam I, Bahrami-Moltagh H, Amerifar M, Zamani N, Hassanian-Moghaddam H. Atypical presentation of COVID-19; an observational retrospective study. BMC Infect Dis. 2020;20(1):870.

23. Ho H-E, Mathew S, Peluso MJ, Cunningham-Rundles C. Clinical outcomes and features of COVID-19 in patients with primary immunodeficiencies in New York City. J Allergy Clin Immunol Pract. 2021;9(1):490-3.e2.

24. Iltaf S, Sr., Fatima M, Salman S, Sr., Salam J-U, Abbas S. Frequency of Neurological Presentations of Coronavirus Disease in Patients Presenting to a Tertiary Care Hospital During the 2019 Coronavirus Disease Pandemic. Cureus. 2020;12(8):e9846-e.

25. Kanji JN, Zelyas N, MacDonald C, Pabbaraju K, Khan MN, Prasad A, et al. False negative rate of COVID-19 PCR testing: a discordant testing analysis. Virology Journal. 2021;18(1):13.

26. Pourbagheri-Sigaroodi A, Bashash D, Fateh F, Abolghasemi H. Laboratory findings in COVID-19 diagnosis and prognosis. Clin Chim Acta. 2020;510:47582. 
27. Zhang Z-L, Hou Y-L, Li D-T, Li F-Z. Laboratory findings of COVID-19: a systematic review and meta-analysis. Scand J Clin Lab Invest. 2020;80(6):441-7.

28. De Carvalho H, Richard MC, Chouihed T, Goffinet N, Le Bastard Q, Freund Y, et al. Electrolyte imbalance in COVID-19 patients admitted to the Emergency Department: a case-control study. Intern Emerg Med. 2021:1-6.

29. Colman J, Zamfir G, Sheehan F, Berrill M, Saikia S, Saltissi F. Chest radiograph characteristics in COVID-19 infection and their association with survival. European Journal of Radiology Open. 2021;8:100360.

30. Huang W, Berube J, McNamara M, Saksena S, Hartman M, Arshad T, et al. Lymphocyte Subset Counts in COVID-19 Patients: A Meta-Analysis. Cytometry Part A. 2020;97(8):772-6.

\section{Figures}

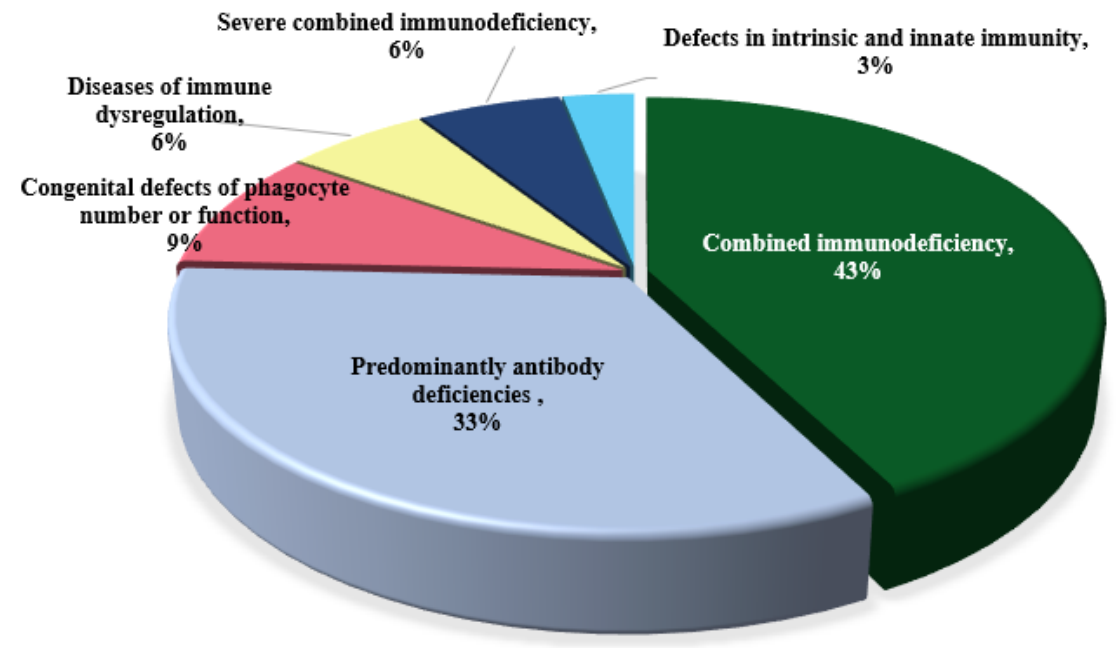

Figure 1

Distribution of IEl groups composed of 33 patients with a diagnosis of SARS-CoV-2 infection. Combined immunodeficiency ( $\mathrm{n}=14)$; predominantly antibody deficiencies ( $n=11)$; Congenital defects of phagocyte number or function ( $n=3)$; Immune dysregulation syndrome ( $n=2)$; Severe combined immunodeficiency $(n=2)$; Defect in intrinsic and innate immunity $(n=1)$. 